\title{
Formalizing Informal Logic
}

\author{
Douglas Walton
}

Centre for Research in Reasoning, Argumentation and Rhetoric

Department of Philosophy

University of Windsor

Windsor, $O N$

Canada N9B 3P4

dwalton@uwindsor.ca

\section{ThOMAS F. GORDON}

Institute of Computer Science

University of Potsdam

August-Bebel-Str.89

14482 Potsdam, Germany

thomas.gordon@fokus.fraunhofer.de

\begin{abstract}
In this paper we investigate the extent to which formal argumentation models can handle ten basic characteristics of informal logic identified in the informal logic literature. By showing how almost all of these characteristics can be successfully modelled formally, we claim that good progress can be made toward the project of formalizing informal logic. Of the formal argumentation models available, we chose the Carneades Argumentation System (CAS), a formal, computational model of argument that uses argument graphs as its basis, structures of a kind very familiar to practitioners of informal logic through their use of argument diagrams.
\end{abstract}

Resumé: Dans cet article nous examinons la mesure dans laquelle des représentations formelles d'argumentation peuvent formuler dix caractéristiques de base de la logique non formelle identifiées dans la littérature de la logique non formelle. En montrant comment la quasi-totalité de ces caractéristiques peuvent être représentées formellement avec succès, nous prétendons que des progrès peuvent être accomplis vers le projet de formaliser la logique non formelle. Parmi les représentations d'argumentation disponibles, nous avons choisi le système Carneades Argumentation (SCA), une représentation de calcul formel d'argument qui utilise des schémas d'argument comme sa base, qui sont très familiers aux praticiens de la logique non formelle qui emploient des diagrammes pour représenter la structure des arguments.

Keywords: informal logic; formal argumentation systems; real arguments; premise acceptability; conductive argument; RSA triangle; relevance; sufficiency. 


\section{Introduction}

In this paper we investigate the extent to which formal argumentation models can handle ten characteristics of informal logic identified in the literature. We show how almost all of these characteristics can be successfully modelled formally, and on this basis we claim that good progress is being made toward the goal of formalizing informal logic. This is to assume that one accepts that these ten characteristics are sufficient, defining characteristics of informal logic. Seen in another way then, our analysis may open the way for further discussion to identifying other defining conditions of informal logic, which may or may not be amenable to formalization.

To begin then, we need to decide what requirements something has to meet to be an informal logic. We take the following ten characteristics of informal logic as our guide. (1) Informal logic recognizes the linked-convergent distinction, (2) serial arguments and (3) divergent arguments. Informal logic includes three postulates of good argument in the RSA triangle: (4) relevance, (5) premise acceptability and (6) sufficiency. (7) Informal logic has recognized the importance of pro-contra (conductive) arguments. (8) Informal logic is concerned with analyzing real arguments. Johnson $(2006,246)$ expressed this characteristic as follows: "[Informal logic] may be seen as a turn toward seeing argument in a real-life setting as opposed to the artificiality of the examples associated with formal deductive logic". There is also a ninth characteristic, (9) the appreciation of the importance of argument construction: "If one is to teach students about real arguments, then it is not enough to focus only on evaluation; one must include the task of argument construction-an emphasis taken from colleagues in rhetoric" Johnson (2006, 248). Argument construction was traditionally called the art of argument invention in rhetoric (Kienpointner, 1997). (10) There is also a tenth characteristic, one that is very important for rhetoric, the notion of audience. Blair (2001, 366) stated that there is general agreement among argumentation scholars that argumentation is a complex social, speech activity involving more than one party, adding "One cannot argue without at least an imaginary audience or interlocutor".

The fulfillment of these particular conditions by a formal system justifies our hypothesis that informal logic can be formalized because, as shown by our survey of the literature in search of definitions of informal logic, although no definition was found which enjoys broad consensus, the ten characteristics identified play a central role in all the proposed definitions. 
Thus a formal model that exhibits all of these characteristics would satisfy all of the proposed definitions, without having to accept any one definition in particular. This however leaves open the possibility that none of the definitions proposed thus far are adequate, leading to continued discussion to search for other characteristics and a new definition. However, in this paper we do not consider if there are any other conditions whose fulfillment allows for a better formalization. Instead of trying to define informal logic ourselves, we limited our task to the question of the amount of progress made toward formalizing informal logic as it is defined in the literature.

The word 'formal', as used in writings on logic and philosophy, can have seven different meanings. One of these meanings, distinguished by Barth and Krabbe (1982, 14-15), is that of a fundamental general term for a concept. For example, one might cite the term 'triangle', which in the Platonic philosophy refers to a general concept of triangularity that is common to all triangles. The second meaning is that of a wellformed formula, for example in a propositional or predicate logic. This meaning is syntactic in nature. The third meaning is that of a formal system, with a set of axioms and inference rules used to derive theorems from the axioms. The fourth meaning refers to formal logic, which is a species of a formal system. A fifth meaning is that of a formal theory, that is, an axiomatization of a theory in a formal logic. A sixth meaning is that of a mathematical structure consisting of sets and operations on the sets. An example would be an algebraic structure. The seventh meaning is that of a formal procedure, for example the kinds of procedures used in court cases.

There are many automated systems to assist with argument diagramming (Scheuer et al., 2010). CAS, however has one of the few argument diagramming tools based on a formal, computational model of argument. CAS is named after the Greek skeptical philosopher Carneades (Gordon and Walton, 2006), and is open source software, available for downloading at http://carneades.github.io/. The point is worth emphasizing that there are formal systems other than calculi for classical logic, and that CAS is a formal, computational model of argument. It is computational, because the model consists of a mathematical structure whose operations are all computable. It is formal, because there is a formal calculus for computable functions (lambda calculus). The rest of this paper presents CAS in more detail and then shows how CAS can be understood as a formalization of informal logic, realizing all of its leading characteristics. 
There are other formal argumentation systems that have been developed in computer science to analyze and evaluate argumentation and that use argument diagrams and other tools comparable to those used in CAS (Besnard et al., 2014). Any one of these systems could be used to formalize argumentation of the kind we have identified as being centrally important for informal logic in our ten characteristics of informal logic. An important property of these systems is that they use argumentation schemes, and although they are quite capable of modeling deductive reasoning and inductive reasoning of certain kinds, like CAS they treat many argumentation schemes as representing forms of argument that are inherently defeasible (Verheij, 2003; Prakken, 2011). Again, like CAS, they formally model the conditions under which an argument can be either supported or defeated by the pro and con arguments in a given case.

One such system ASPIC+ (Modgil and Prakken, 2014), is built around the notion of defeasibility attributed to (Pollock, 1995), that is based on a distinction between two types of argument attacks called undercutters and rebutters. ASPIC+ is based on a logical language consisting of a set of strict and defeasible inference rules used to build arguments from a knowledge base of premises that can be combined with the inference rules to generate a sequence of argumentation in the form of a directed graph leading to an ultimate conclusion in a tree structure (Prakken, 2011). The logical system DefLog (Verheij, 2003, 2005) uses an argument diagramming tool called ArguMed that can be used to analyze and evaluate defeasible argumentation. This logical system is built around two connectives called primitive defeasible implication and dialectical negation.

Either of these systems can be used to do many of the same tasks that CAS will be shown to do in this paper. There are also many more resources available in artificial intelligence that could also be applied to the task of formalizing informal logic, in somewhat different ways, by using comparable tools that would produce comparable results in modeling typical tasks of argument evaluation and analysis carried out in informal logic.

\section{The Carneades Argumentation System}

CAS formalizes argument graphs, as bipartite, directed graphs, consisting of argument nodes linked to statement nodes. The CAS User Manual for the latest version can be found at 
https://carneades.github.io. A graph is defined mathematically as a set of vertices, also called points or nodes, and a set of edges, also called lines or arcs. The graph is called a directed graph if every pair of its elements is an ordered pair. CAS argument graphs model relationships among arguments and statements (propositions). CAS can be used for argument construction as well as argument reconstruction. In the species of argument reconstruction familiar in informal logic, arguments are identified and analyzed, typically using an argument diagram, from source documents, for example court documents or social or political commentaries of the kind found in magazines newspapers, or on the Internet. In argument construction, also called argument invention (Walton and Gordon, 2012), arguments represented in an argument graph can be extended to build up new arguments constructed from a knowledge base consisting of evidence and facts. Argument nodes are of two types, pro and con. Carneades argument diagrams (or maps) visualize the arguments found in such sources as argument graphs. Conceptually it is important to distinguish such visualizations from the underlying mathematical structure being visualized. Argument graphs can be visualized in different ways and levels of abstraction, for different purposes.

Argument graphs model inferential relationships among arguments and statements. An argument graph is a bipartite, directed, labeled graph, consisting of statement nodes and argument nodes connected by premise and conclusion edges. Formally, an argument graph is a 4-tuple $\langle S, A, P, C\rangle$, where $S$ is a set of statement nodes, $A$ is a set of argument nodes, $P$ is a set of premises, and $C$ is a set of conclusions. The 4-tuple does not model a single argument, but rather a set of arguments, a whole argument graph. A single argument is a subgraph of the argument graph, where the subgraph is a tree (no cycles) and none of the leaves of the tree are issues but rather assumed to be true or false or rejected or accepted by the audience.

To see an example, look ahead to figure 3 . The statement nodes are shown as the rectangular text boxes in the figure containing statements. The argument nodes are the two circles containing the plus signs. The two premises are the statements in the text boxes on the right. The conclusion is the statement that the death penalty is wrong.

Let $L$ be a propositional language, consisting of a set of propositional letters. Each statement node in $S$ is labeled with a propositional letter in the language $L$. Each argument node in $A$ is a structure $\langle i d, s, d\rangle$, where $i d$ is some unique term naming the argument and $s$ is a Boolean value which is true if the argument 
node is strict and false if it is defeasible. $d$ is also a Boolean value, representing the direction of the argument, which is true if the argument is pro its conclusion and false if it is con its conclusion. The premises and conclusions of an argument graph represent the edges of the graph, connecting the statement and argument nodes.

Each premise in $P$ is a structure $\langle s, a, p\rangle$, where

$$
\begin{array}{ll}
1 & s \in S, \\
2 & a \in A, \\
3 & p \text { is a Boolean value denoting the polarity of the } \\
\text { premise, i.e. positive or negative. If } p \text { is true, then the } & \\
\text { premise is positive, otherwise it is negative. } & \text { Each conclusion in } C \text { is a structure }\langle a, s\rangle, \text { where } \\
1 & a \in A, \text { and } \\
2 & s \in S
\end{array}
$$

Every argument node has exactly one conclusion. That is, for every argument $a \in A$ there exists exactly one $(a,\rfloor \in C$. An argument node may have zero or more premises. No two argument nodes in an argument graph have the same identifier.

Argument graphs are evaluated, relative to audiences, to determine the acceptability of statements in a stage (Gordon and Walton, 2009). Audiences are modeled as a set of assumptions and an assignment of weights to argument nodes. A literal is either a propositional variable or its negation. Where $L$ is a propositional language as defined above, an audience is a structure <assumptions, weight $>$, where assumptions $\subseteq L$ is a consistent set of literals assumed to be acceptable by the audience and weight is a partial function mapping arguments to real numbers in the range $0.0 \ldots 1.0$, representing the relative weights assigned by the audience to the arguments (Gordon and Walton, 2011). More recently we have found a method to evaluate cyclical argument graphs in a way compatible with the semantics of the original system, via a mapping from argument graphs to Dung abstract argumentation frameworks (Dung 1995), similar to the mapping of ASPIC+ (Prakken 2010; Bin and Prakken, 2012).

In (Gordon, Prakken \& Walton, 2007) the acceptability of statements was defined directly, via a set of mutually recursive functions, but only for acyclic argument graphs. Conflicts between pro and con arguments are resolved using proof standards. The proof standard of a statement determines how much proof is required for the statement to be taken as 
acceptable (presumably true). The proof standard is used by the formal model argument to determine the acceptability of the statement. Proof standards have a legal flavor, and the notions of proof standards and burdens of proof modeled in CAS are motivated by an interest in legal applications. Several legal standards of proof exist, for example the preponderance of the evidence standard, also known as the balance of probabilities, the standard applicable in civil cases. The preponderance standard is met by the proposition at issue if its pro arguments are stronger than its con arguments, no matter how much stronger they may be. The beyond reasonable doubt standard, the highest standard used in Anglo-American jurisprudence, and the standard applicable in criminal law, requires that the arguments supporting the claim must not be amenable to any opposing arguments from critical questions that can leave any doubt open on whether the claim is acceptable. This standard does not require a proof to show that a claim is true with absolute certainty. It is not a standard of beyond all doubt. It only needs to be strong enough to overcome a reasonable doubt that can be raised by arguments or questions put forward by the defense. The clear and convincing evidence standard, lying between the other two standards, is higher than the preponderance of the evidence standard but not as high as the beyond reasonable doubt standard. These are just some examples of standards of evidence that are applicable in legal argumentation. The standards modelled in CAS are defined more precisely in section 6, where the question of how CAS models argument sufficiency is raised.

CAS also formalizes argumentation schemes. Schemes can be used to construct or reconstruct arguments, as well as to check whether arguments are "valid", i.e. whether they properly instantiate the types of argument deemed normatively appropriate for the type of dialogue. In CAS, argument evaluation is the process of critically assessing arguments by four means: (1) revealing implicit premises, (2) validating whether the arguments are formally correct, by instantiating accepted argumentation schemes, (3) asking critical questions appropriate for a scheme, and (4) determining which claims are acceptable, taking into consideration the assumptions of the audience and its assessment of the relative weights of conflicting pro and con arguments. The first three of these tasks can be accomplished by comparing the argument with its argumentation scheme.

You can view a list of the argumentation schemes available in CAS and choose which one to apply. For example if 
you type in the term 'practical-reasoning', CAS will display the scheme containing this term, as shown below. A1, A2, . ., An represent actions and S1, S2, ..., Sn represents "states" which we can think of as circumstances of a case. The argument identifier is represented by an id.

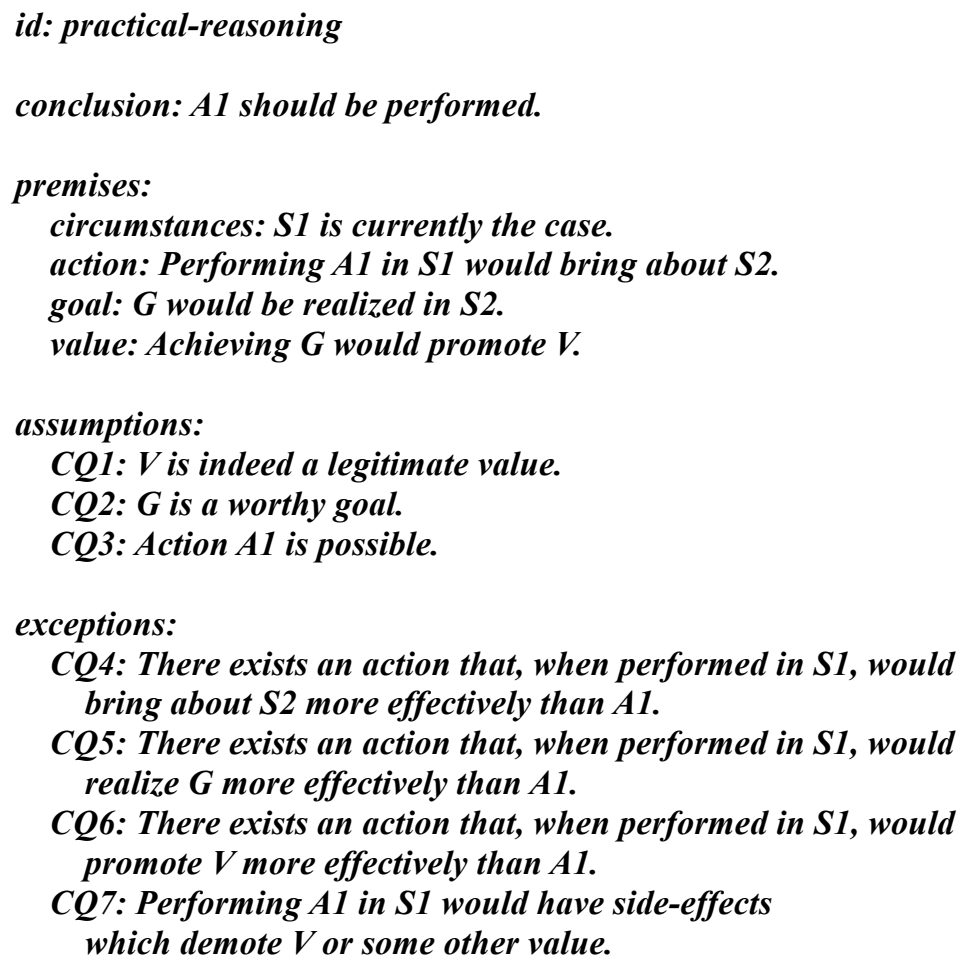

Whenever a scheme is selected, the form will be customized to include premises and exceptions fields for the chosen scheme. The roles of the premises and exceptions will be modified to match the selected scheme. If no scheme matches the argument in a given text you are trying to reconstruct, you can put in your own scheme, put in no scheme, or classify the type of argument under a more general scheme such as deductive modus ponens or defeasible modus ponens.

CAS is capable of representing instances of any kind of argumentation scheme, whether deductive, inductive or defeasible, such as argument from expert opinion. The conclusion of a defeasible argument is only presumptively true. Defeasible arguments can be defeated by counterarguments of various kinds. CAS has mainly been tested on examples of legal argumentation, but it is open domain software, meaning that it can be applied in other contexts of use, including everyday conversational argumentation. 


\section{Single, linked, convergent, serial and divergent arguments}

The first step in understanding an argument diagramming system is to see how it represents linked and convergent arguments. A linked argument is one where the two (or more) premises go together to support the conclusion. A convergent argument is one where each premise (or group of premises) function together to support the conclusion.

As types of structures that appear in argument diagrams, informal logic recognizes five kinds of arguments, single, linked, convergent, serial and divergent. In the simplest kind of case, called the single argument, there is only one premise and one conclusion (Walton, 1996, 84). The following example of a single argument is cited in (Walton, 1996, 84).

Webb was promoted to vice president, therefore she will move to Pittsburgh.

How this example is represented by CAS is shown in figure 1, where the plus symbol in the argument node indicates that this is a pro argument. CAS uses a minus sign in the argument node to indicate a con argument.

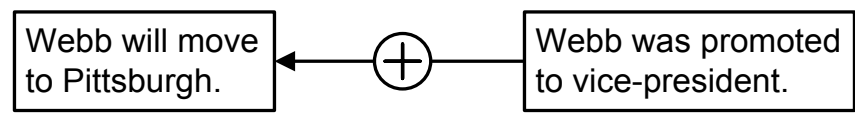

Figure 1: Single argument in Carneades

A linked argument is an argument that has more than one premise, and its premises function together to give support to the conclusion (Walton 1996, 85). According to (Copi and Cohen, 1990, 20) in a linked argument with two premises, each premise supports the conclusion through the mediation of the other so that neither supports the conclusion independently. One of the examples given in (Walton, 1996, 87) is an instance of practical reasoning.

My goal is to get to Leiden, taking the maaldrift is the way to get to Leiden, therefore I should take the maaldrift: 


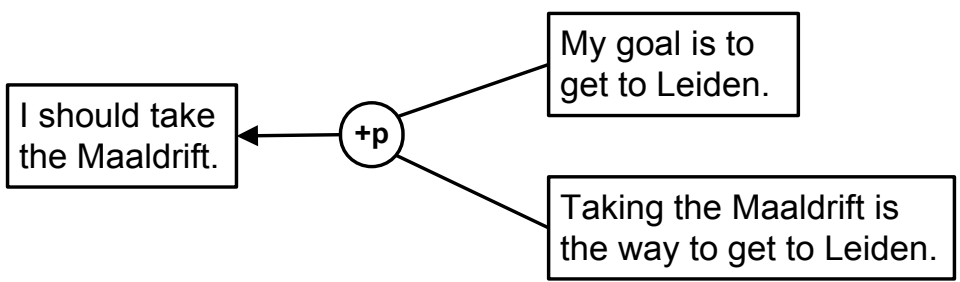

Figure 2: Linked argument in Carneades

The letter $\mathrm{p}$ in the circular argument node stands for the argumentation scheme for practical reasoning. The plus sign in the node indicates that the argument fitting the scheme is a pro argument. The practical reasoning scheme (in its bare-bones form) represents the following form of argument: I (an agent) have a goal $G$; carrying out action $A$ is the way to obtain $G$; therefore I should carry out $A$.

In a convergent argument each premise gives independent support to the conclusion. An example (paraphrased from Copi and Cohen, 1990,22) has the conclusion that the death penalty is wrong. The two premises given to support this conclusion are (1) there is not enough evidence to show that the death penalty is a deterrent and (2) there are better and more effective ways to deal with violent crime.

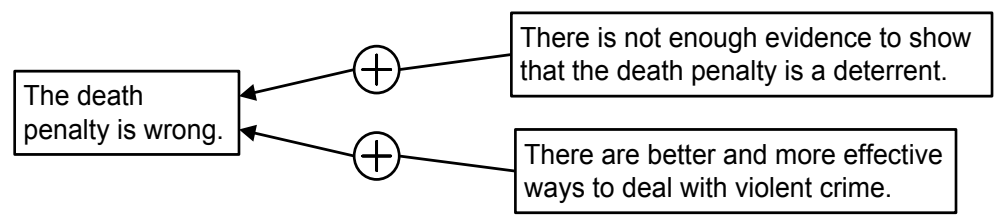

Figure 3: Convergent argument in Carneades

As indicated in figure 3, convergent arguments are represented using multiple argument nodes, instead of using a bracketing line to join the premises together and then drawing the arrow from the bracketing line to the conclusion.

In a serial argument, often called a chain argument, the conclusion of one argument also functions as a premise in a second argument, and so forth, forming a chain of arguments. Typical Carneades argument maps display lots of chained arguments, as the example in figure 4 shows. 


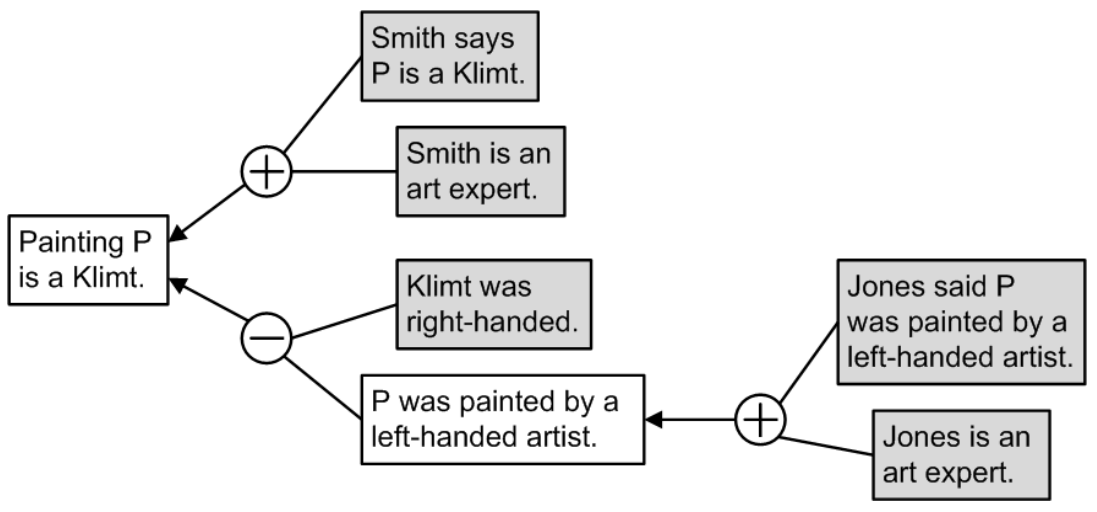

Figure 4: CAS argument map with a chained argumentation structure

This map includes an example of a con argument, indicated by the minus sign in the node at the bottom left. It also shows a chained argument. The linked argument at the right, an argument from expert opinion, leads to the conclusion that the portrait showed evidence of being drawn by a left handed artist. This proposition, in turn, serves as a premise in the linked con argument rebutting the conclusion that painting $\mathrm{P}$ is a Klimt. This example can also be used to illustrate some points about how CAS evaluates arguments.

The top argument is also an argument from expert opinion. Note that although argumentation schemes are not displayed in figure 4, they are represented in the underlying data model of the argument graph. Note that the scheme for argument from expert opinion could be applied to two arguments, in each instance showing that there is a missing premise. For example the implicit premise 'Attributing painting $\mathrm{P}$ is in the domain of art' can be added to the top argument.

Figure 4 can also be used to illustrate how an argument graph can be evaluated by the computational model. Statement and argument nodes are evaluated to be one of three values: in, out, or undecided. In figure 4, the five in nodes representing statements that the audience accepts, are shown filled with gray (green normally, but since we can't use color here, they are shown in gray). Statements that the audience rejects, out nodes, are filled with a red color, but none of these are shown in this example. The two statements that the audience neither accepts nor rejects, undecided nodes, are shown with a white background. The values of the remaining statement nodes are computed using proof standards and the weights assigned by the audience to the argument nodes. Whether a proposition is in or out 
initially is determined by whether or not the audience accepts it. In figure 4, the audience has accepted all the five propositions shown in boxes with gray backgrounds. The only propositions the audience does not accept (at least so far) are the two shown with white backgrounds. Given this information about what the audience accepts or does not, CAS can calculate whether the argument justifies acceptance of its conclusion or not.

In this instance, both premises of the top linked argument are accepted, and so the conclusion should also be accepted, assuming this argument meets its standard of proof and is therefore sufficient to prove the conclusion. Just on this basis alone, the conclusion should be shown as accepted by showing it with a gray background. But this argument is not sufficient to justify acceptance of the conclusion that the paining is a Klimt, because the con argument at the bottom also has to be taken into account. So we have to look at the bottom argument. The bottom premise, the statement that $\mathrm{P}$ was painted by a left handed artist, is shown as 'not accepted' by the audience. But it is supported by the con argument showing at the right, a linked argument that has both premises accepted. Therefore CAS automatically computes that proposition ' $\mathrm{P}$ was painted by a left-handed artist' is justified, and colors it gray. So now we have a pro argument with all its premises accepted pitted against a con argument with all its premises accepted. Which one wins the battle of the experts? This will depend on two factors: (1) the standards of proof assigned each of the two arguments, and a weighing of the comparative strength of the two arguments. Later it will be shown how this is done.

A divergent argument (Walton 1996, 91) is one in which two separate conclusions are each supported by the same premise. The following example from (Walton, 1996, 91) was originally taken from a Sherlock Holmes story. Smith is not the murderer, therefore (1) Robinson had nothing to do with the crime, and (2) Lady Gregg's display of grief was merely a tactic to cover up the finding of the revolver. Figure 5 shows how divergent arguments are modeled in Carneades.

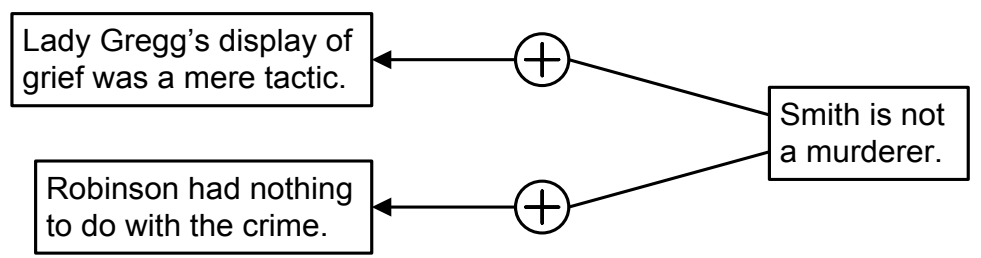

Figure 5: Carneades argument map of a divergent argument 
In CAS, premises and conclusions are relations between argument nodes and statement nodes. The same statement node can be a premise or conclusion of more than one argument node. Figures 3 and 5 provide illustrations. In Figure 3, the statement node for "The death penalty is wrong" is a conclusion of two argument nodes, with different premises. In Figure 5, the statement node for "Smith is not a murderer" is a premise of two different argument nodes, with different conclusions.

Finally in this section, we reply to an objection. Can CAS handle the following kind of convergent argument, where the premises support the conclusion independently? The conclusion is "Foreigner X can communicate in English" and the two premises are "X has CAE" and "X has CPE", where CAE is the Certificate in Advanced English and CPE is the Certificate of Proficiency in English. Both premises treated separately as single arguments support the conclusion, but since CPE is a much weaker and more restricted test of English linguistic ability than $\mathrm{CAE}$, taken together as a convergent argument they do not support the conclusion independently. The influence of CAE is marginal, if it has any at all. Can CAS allow for representation of the argumentation structure in this kind of case?

CAS can handle this example, since it does not assume that arguments are independent. Argument weights are not automatically aggregated (e.g. summed) by the model. Only the strongest pro and con arguments are compared against each other by the proof standards. Arguments can be aggregated manually and then weighed again, but this weighing is done manually by the audience, not the formal model, and it is the audience's responsibility to not count common features of the arguments multiple times.

\section{The RSA Triangle}

Blair $(2012,87)$ wrote that when he and Ralph Johnson first wrote their textbook Logical Self-defense (first edition, 1977), they used the relevance sufficiency acceptability (RSA) triangle to determine whether an argument is a good one. According to the RSA principle, an argument is a good one if its grounds (or premises) singly or in combination meet three criteria. First, the premises have to be individually acceptable. Second, taken together the premises have to be sufficient to support the claim that is the conclusion of the argument. Third, the argument needs to be relevant as a support for the conclusion. Blair (2012, 88 ) wrote that he and Johnson had the RSA criteria in mind as a 
replacement for what he called the traditional soundness criterion, which maintains that a good argument is a sound argument, and a sound argument is one that is deductively valid and has true premises.

Formal argumentation systems of the kind currently being developed in artificial intelligence use argumentation schemes to model defeasible forms of argument that are subject to critical questioning. Such systems evaluate an argument as a good one or not on a balance of pro and con considerations. The model used is a dialectical one in which an argument that is a good one shifts the burden of proof to a critic or opponent to provide reasons for not accepting it. In particular CAS evaluates an argument in relation to whether the premises of the argument are accepted by the audience to whom the argument was directed, and the appropriate inferential link joining the premises to a conclusion, generally represented as an argumentation scheme, transferring acceptance from the premises to the conclusion.

\section{Acceptability}

A simple example of how CAS uses the device of an audience to evaluate arguments is shown in figure 6. Let's consider a case of a deliberation dialogue where Bob has acid indigestion and is considering taking a medication $\mathrm{M}$ to treat his condition. He is examining the pros and cons in trying to reach a reasonable decision on whether he should take $\mathrm{M}$ or not. He has a bottle of $\mathrm{M}$ handy and is trying to decide what to do. Bob might reason as shown in figure 6. Let's say that Bob is thinking that if he takes $\mathrm{M}$, it would relieve his acid indigestion. He tells his partner Alice, "I should take M". Using the argumentation scheme for value-based practical reasoning presented in section 2 to fill in some implicit premises, Bob's reasoning could be represented in CAS as shown in figure 6.

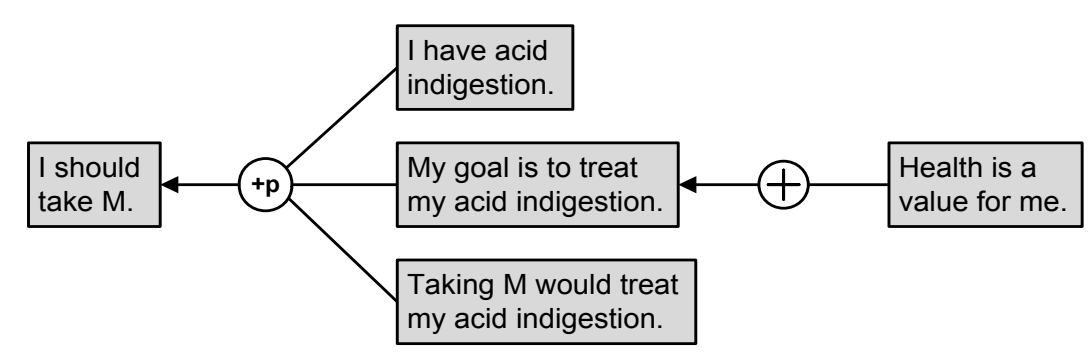

Figure 6: First argument diagram for the indigestion example 
Notice that this way of modeling the argument follows the standard way of representing value-based practical reasoning (Atkinson, Bench-Capon and McBurney, 2006). On this model, the value of health is shown as supporting the agent's goal of treating his acid indigestion. When premises are put forward to support a claim, in an example of this sort, the normal premises that fit the scheme are represented as assumptions rather than exceptions. Critics only need to question assumptions, after which they act like ordinary premises which must be proved by the proponent of the argument. (This is how assumptions differ from exceptions, where the critic has the burden of proof (evidential burden) and must put forward an argument proving the exception.) So the four premises of the practical reasoning argument are displayed in text boxes with gray backgrounds, indicating audience acceptance. Since the argument fits the scheme for value-based practical reasoning, let's also assume that the audience accepts the argument strongly enough to meet the required standard of proof to prove the conclusion 'I should take M' shown at the left of figure 6. So far then, the outcome is that CAS replaces the white background of the conclusion box with a gray background.

But let's continue the dialogue a little further. Suppose that Alice reads the small print on the bottle, and sees that it warns that taking $M$ could have a side effect of gastrointestinal bleeding. Here the side-effects critical question CQ7, shown in the CAS scheme presented in section 2, comes into play. CQ7 is listed as an exception, and it says that performing A1 in S1 would have side-effects which demote V or some other value. So how could one proceed further to represent the structure of the new sequence of argumentation? By raising a critical question, Alice's next move in the dialogue in effect poses a counterargument, and therefore we have to examine how CAS treats critical questions and counterarguments.

In the most recent versions of CAS, exceptions are treated as Pollock-style undercutters. (Pollock, 1995) distinguished between two kinds of counter-arguments he called rebutting defeaters and undercutting defeaters (often referred to as rebutters and undercutters). A rebutter gives a reason for denying a claim by offering reasons to think it is false (Pollock, 1995, 40). An undercutter attacks the inferential link between the claim and the reason supporting it by undermining the reason that supported the claim. CAS has three ways in which one argument can attack and defeat another, based on this distinction. A rebutter is an argument that attacks the conclusion of a prior argument by presenting a reason to think the conclusion is false. An undercut- 
ter attacks the argument link between the premises and the conclusion, for example by asking a critical question pointing to an exception to the holding of the argument. For example, an argument that fits the argumentation scheme for argument from practical reasoning could be defeated by the asking of any one of the critical questions shown in section 2 .

\section{Sufficiency}

CAS is built around the idea of modeling sufficiency by using proof standards to aggregate pro and con arguments (Gordon, Prakken and Walton, 2007). The proof standard of a statement determines how much proof is required for the statement to be deemed acceptable (presumably true). The proof standard is used by the computational model of argument to compute the acceptability of the statement (Gordon and Walton, 2009). As promised in section 2, it will now be shown how four proof standards employed in CAS are defined.

The conclusion of an argument is in (acceptable) if it has been accepted by the audience or it satisfies the proof standard appropriate for the type of dialogue. The standard of dialectical validity (DV) is met if at least one pro argument is in and no con argument is in. The preponderance of evidence (PE) standard is met if at least one pro argument is in that weighs more than any in con argument. The clear and convincing evidence (CE) standard is met if the preponderance of evidence standard is met and, in addition, the difference between the strongest in pro argument and the strongest in con argument is above a certain threshold.

The beyond reasonable doubt (BRD) standard is met if the clear and convincing evidence standard is met and, in addition, the weight of the weakest in con argument is below a certain threshold. The default proof standard is preponderance of the evidence, and for most applications this proof standard is sufficient. Note that the preponderance of evidence standard is met whenever the dialectical validity standard is met. The preponderance of evidence, clear and convincing evidence and beyond reasonable doubt standards are ordered by the amount of proof required, with beyond reasonable doubt requiring the most proof. Whenever one of these standards is met, all of weaker standards are also met.

Next we use the indigestion example to show how CAS models the notion of argument sufficiency using proof standards. Basically, an argument is sufficient to prove its conclusion 
if it fulfills its burden of proof required to prove the conclusion. Burden of proof is set by the standard of proof appropriate for the argument, as determined by the user when the user puts one of the four standards of proof defined above. So far, as shown in figure 6 , all three premises in the present example have been accepted and are thus in, and so the conclusion is also shown in a gray box. But in figure 7, Alice's critical question is modeled as an undercutter. In CAS, an undercutter is modeled as a secondary argument that attacks the original argument.

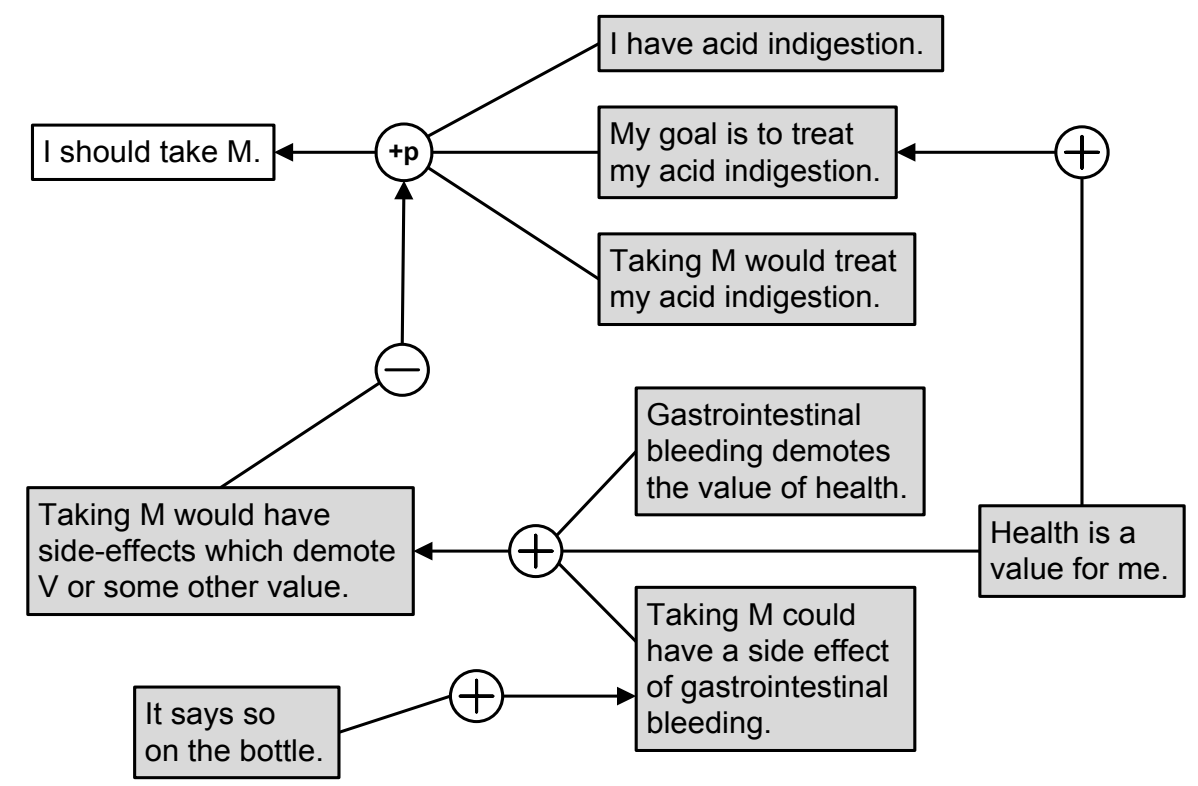

Figure 7: Second argument diagram for the indigestion example

In figure 7, CQ7 is represented as a con argument with a premise stating that taking $M$ would have side effects that demote $\mathrm{V}$ or some other value. The counterargument is shown not as attacking any premise or the conclusion of the original practical reasoning argument used by Bob, but instead its argument node is joined by an arrow to the argument node above it representing the scheme for practical reasoning. This example shows the distinctive way in which CAS models an undercutter as one argument attacking another. To see how CAS models this kind of situation, we have to see how it distributes the burden of proof in a dialogue when critical questions corresponding to an argumentation scheme are asked. 
How strongly the audience accepts the argument can be represented numerically in CAS. This feature can be used to break deadlocks between an opposed pair of arguments. These numerical weights are used by some proof standards to evaluate arguments.

The approach taken in CAS to the problem of determining how the burden of proof should be distributed is as follows (Gordon and Walton, 2011). The burden of production is distributed by dividing premises into different types: evidence for ordinary premises and assumptions (once challenged) must be produced by the proponent of the argument, while evidence for exceptions must be produced by the respondent. There are two kinds of burden of proof. One is the so-called burden of persuasion set at the opening stage of dialogue. The burden of persuasion is allocated by assigning the appropriate proof standard. The other is the so-called burden of production, sometimes also called the evidential burden, which can shift from side to side as the dialogue proceeds. CAS allows the burdens of production and persuasion to be allocated separately to either the proponent or the respondent and modified during the course of the dialogue. The initial allocation of the burden of production is regulated by the premise types of the argumentation scheme applied. The values (in, out, undecided) of the nodes in the argument graph are computed by the model once the user has put in whether the audience accepts the premises or not, unless a critic questions the assumption (No reasons or arguments need to be put forward to question assumptions, in contrast to exceptions). Then the argument is evaluated using the standards of proof.

Looking back to figure 7, let's see how CAS evaluates the argument once Alice's move of putting forward her critical question has been made. First it needs to be recalled that CQ7 is classified in the scheme for practical reasoning as an exception. This means that the burden of proof is on Alice to back up her premise that taking $\mathrm{M}$ would have side effects which demote $\mathrm{V}$ or some other value by offering an additional argument to support this claim. If she fails to provide such an argument, her critical question will fail to shift the burden of proof back onto Bob's side. But as the reader can see by looking at figure 7, Alice has provided a pro-argument supporting her exception. This pro argument has three premises, one of which is the reused statement that health is of value for me. Note that CAS can reuse a premise in a different argument.

Not only that, Alice presents another pro argument to back up her premise that taking $\mathrm{M}$ could have a side effect of gastrointestinal bleeding. Assuming that all the premises of Alice's 
counter-argument are accepted by the audience (they are assumptions), Alice has fulfilled her burden of proof to support her assertion about the side effects of taking M. Hence CAS will automatically remove the gray background from the text box containing the conclusion that Bob should take $\mathrm{M}$. In other words, Alice's critical question has undercut Bob's original argument based on practical reasoning, and has thereby shifted the burden of proof back onto Bob side to make another move. Bob could respond by providing additional arguments to back up his original argument, or by attacking Alice's counterargument in some appropriate way.

\section{Relevance}

Ballnat and Gordon (2010) provided a method of argument construction for CAS, and Walton and Gordon (2012) have shown how the method can be applied to arguments of the kind that are of central interest for informal logic. To apply the method, the arguer needs to build his argument with the goal of getting the audience to accept some designated proposition that represents his thesis to be proved by basing his arguments on premises that his audience either accepts or can be led to accept by argumentation. If the audience accepts the premises, and if the argument is structurally correct by application of argumentation schemes, the audience will also need to accept the conclusion, or give arguments to show why it should not. To use the system, an arguer provides input on which premises the audience has accepted or not. Then it searches for a path leading from these premises (along with others) to the ultimate probandum. When it finds such a path, it tells the user which premises remain to be accepted. If it finds no such path, it gives advice on what positions could be useful to work towards finding a path.

Relevance of arguments has not yet been formally modeled in CAS, but here we can briefly outline how this research project could plausibly be carried out, based on some previous work in the informal logic area. One important point (Walton, 2004) is the argumentation schemes and their matching critical questions can, in many instances, be used to determine whether one argument is relevant to another, or whether a question or a statement is relevant to an argument. But the problem is that a single argumentation scheme by itself is very often not enough to determine relevance. The reason is that in typical cases arguments are chained to each other, the conclusion of one argument being a premise in the next. Hence proving an argument is rele- 
vant to some ultimate claim representing the issue, the conflict of opinions in the case at issue, requires a model that can show how the argument links up through a series of successive arguments moving toward the ultimate claim. Analyzing relevance in such cases requires building an argument diagram, a graph structure showing a sequence of inferences from premises to conclusions where the sequence ultimately concludes in an endpoint.

According to the analysis of relevance in argument given in (Walton, 2004), relevance needs to be defined and evaluated in a tree structure comparable to argument graphs in CAS. There needs to be a central claim, often called an ultimate probandum in law, at the root of the tree. This framework follows the classical stasis theory well known in rhetoric (Hohmann, 1989; Freeman, 1998; Tindale, 1999). Let AG be an argument graph containing a statement node, $\mathrm{C}$, for the claim. It is a conjecture made in (Ballnat and Gordon, 2010) that an argument node, A, in $\mathrm{AG}$ is relevant to $\mathrm{C}$ if and only if there is a path from $\mathrm{A}$ to $\mathrm{C}$ in AG. Many examples of relevance, both in legal and ordinary arguments, are provided in (Walton, 2004). Although CAS could turn out to be an excellent system for modeling relevance of this kind, so far the project of carrying out such this research task has not yet begun.

Our proposed model of relevance, determined by the existence of a path between the argument and claim in an argument graph, seems plausible to us but remains a project for future work. Unfortunately, we have to admit that this section devoted to the topic of relevance is very short and preliminary. We have not yet attempted to build a model of relevance. It is a centrally important topic of research for argumentation studies, but at the same time it is a highly contested and slippery topic that is hard to say anything very useful about in a short space. The lack of such a model of relevance is the main reason why we do not claim to have yet modeled all the characteristics of informal logic, but only to have made considerable progress towards this worthy goal.

\section{Conductive Arguments}

We take conductive arguments to be the same as pro-contra arguments. Whatever term you choose, the characteristic of them as a class is that they need to be evaluated by taking into account both the arguments for (pro) some contested claim as well as the (contra) arguments against it, and weigh the one side against the 
other. The term 'conductive argument' is taken to have been coined by Wellman (1971), but actually the way the word is used currently in informal logic is different from the narrower meaning of it given by Wellman. Wellman defined conductive reasoning as meeting four requirements $(1971,52)$. (1) It is about a conclusion in some individual case. (2) It is drawn inconclusively. (3) It is drawn from one or more premises about the same case. (4) It is drawn without appeal to other cases. Amplifying the fourth point tells us as well that the most striking feature of all the examples of conductive reasoning he has given is that they all deal with particular cases. This definition clearly excludes arguments from analogy as fitting under the conductive category, since arguments from analogy compare two different cases. However, this restriction is widely ignored in current discussions of conductive argument. Argument from analogy is a very important kind of argument for informal logic, on our view. Much then depends on whether we stay with Wellman's meaning of the term or use it a broader way to refer to all pro-contra argumentation. This broader way does not exclude deductive arguments. A deductive argument rebuts any opposing defeasible argument. Opposing pro and con deductive arguments are also possible, but cannot be in simultaneously unless the statements accepted by the audience are inconsistent.

Wellman tells us that there are three patterns of conductive reasoning. The first is one where a single reason is given for the conclusion. He cited this example: "You ought to help him for he has been very kind to you" $(1971,55)$. This would be the single type of argument, of the four types classified above. The second one is where several reasons are given to support the conclusion. He cites this example: "You ought to take your son to the movie because you promised, and you have nothing better to do this afternoon" $(1971,56)$. This would be a convergent argument. The third one draws the conclusion from both positive and negative considerations. He cites this example: "Although your lawn needs cutting, you want to take your son to the movies because the picture is ideal for children and will be gone by tomorrow" $(1971,57)$. The third pattern shows the paradigm pro-contra feature of conductive arguments.

The last example can also be classified as a convergent argument, but has an additional feature of interest. It is associated with the "balancing" notion of weighing the arguments on both sides of a disputed issue. This notion is one that many in the informal logic community have found so appealing while others dismiss is it as metaphorical (Blair and Johnson, 2011). This balance notion of deciding an issue by weighing one side 
against the other has also been found highly appealing in law, but there too, others have strongly criticized it as an inadequate substitute for deciding cases on the legal rules and the facts of a case (McFadden, 1988).

Either of these arguments can be modeled by CAS, and that may remove some of the doubts about pro-contra argument on the ground that they are merely metaphorical. CAS models it using the pro-contra feature, but in a different way than the arguments that McFadden objected to. He objected to it as a balance of interests, or as a balancing of factors on either side of a disputed issue. But CAS models it as a balance between opposed arguments. Carneades can map the lawn example as shown in figure 8.

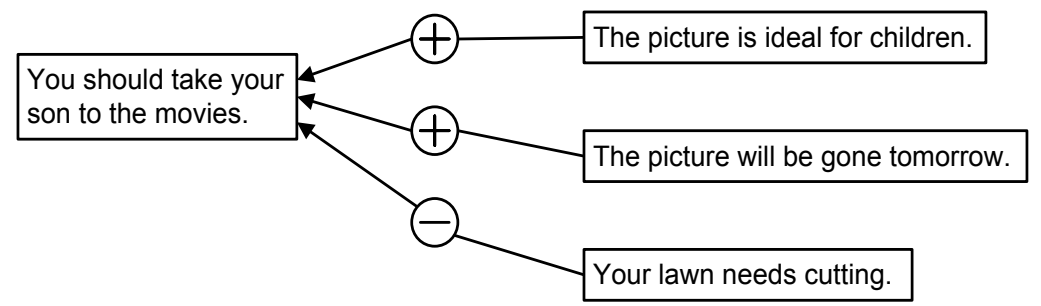

Figure 8: CAS argument map of Wellman's lawn example

As shown in figure 8, the two pro arguments are "balanced" by the con argument, meaning that all three arguments are "good" arguments that carry some evidential weight even though none of them individually, nor any subset of them, is decisive in proving or disproving the conclusion. Should the conclusion be acceptable (in) or not? Even though there are two pro arguments against one contra argument, the number of arguments is not the deciding factor. What is the deciding factor is the audience. Let us presume the audience has accepted all three of the premises. Let's assume that family values outweigh home care values. Then the two pro arguments, taken together, should prevail over the contra argument.

Adler (2013) argued that conductive arguments, as they are commonly characterized, are impossible and therefore can't exist. According to Adler, their property of non-conclusiveness makes conductive arguments impossible, backing up this attack by pointing out that Wellman never provided any definition or explication of 'conclusiveness'. Blair (2013) responded by arguing that Adler's refutation of conductive arguments is based on a misreading of the term 'non-conclusive' and that therefore his dismissal of conductive argumentation fails. Much depends in 
this issue on what 'conclusive' and 'inconclusive' should be taken to mean.

The word 'conclusive', as applied to arguments, is slippery and ambiguous. In one sense, it refers to the drawing of a conclusion from a set of premises implying that this particular conclusion has now been selected so that other conclusions no longer need to be considered. This meaning of the word does not rule out that the argument may have to be revised at some point in the future, if a different conclusion is arrived at that will replace the previous one. This meaning of the term can also be called detachability, implying that the conclusion can now be detached from the argument used to support it or arrive at it. The idea is that it can now be seen as separately acceptable in its own right, and used as an acceptable premise in a new argument. In a different meaning of the word, to say that an argument is conclusive means that its conclusion has been proved to such a degree of certainty that there will or should be no need to withdraw it in the future. It is easy to confuse the two meanings, and it is not certain which of them should be taken as the best meaning of the term for logic and philosophy.

The first meaning appears to be a narrower one, whereas the second one seems to be more important for the field of philosophy, where there is always a quest to look for an argument or proof that is conclusive. The basic idea behind the second meaning is that a conclusive argument leaves no room for doubt that its conclusion is true. But this notion is philosophically controversial, because there is a school of thought in epistemology called skepticism that claims that no real argument is so strong (conclusive) that no room at all for doubting its conclusion is left. But even this view can maintain that an argument is conclusive if it removes enough room for doubt so that the conclusion can be accepted as strong enough to eliminate the need for further pro-con argumentation.

Deductive arguments are not conclusive, in the sense that a deductive argument proves its conclusion beyond all doubt. Deductive arguments can be attacked in two ways. First, a counterargument can be found that shows that one (or more) of the premises of the deductive argument does not hold. Second a deductive con argument can be brought forward based on premises more strongly accepted than the first argument, and attack it. The first form of con argument is called a premise attack and the second is called a rebuttal.

Conductive arguments, judging by the examples of Wellman analyzed in this paper, certainly do seem to be defeasible, and if defeasibility implies inconclusiveness, in Adler's sense of 
the term, then Blair is certainly right to reject Adler's rejection of conductive arguments. One of the merits of such arguments is that they are distinctly different from deductive arguments precisely because they have the property of defeasibility. In any event, an interesting issue is opened concerning what meaning 'conclusiveness' should be taken to have. CAS can throw some light on this issue insofar as it relates to burdens and standards of proof.

The default proof standard in CAS is preponderance of the evidence, and this standard is met whenever the dialectical validity standard is met. Arguments can be weighed numerically in CAS or not, and if they are not weighed, the dialectical validity and preponderance of evidence standards give the same results. The preponderance of evidence, clear and convincing evidence and beyond reasonable doubt standards are ordered from the weakest to the strongest. When one is met, all of weaker standards are also met.

It should be mentioned in passing that CAS also allows argumentation to have an opening stage, an argumentation stage and a closing stage, bringing in the possibility of a procedural side to the issue of how to determine conclusiveness of an argument. From a procedural point of view, an argument may be viewed as conclusive if no further evidence or arguments may be put forward in the dialogue, according to the procedural rules (protocol) governing the dialogue. This depends on how the closing stage is reached in any given case, depending on closure rules. However, this is merely an aside, showing another of the many sides to the controversial question of conclusiveness. Here the main justification of our claim that CAS can model the concept of argument sufficiency rests on its use of proof burdens and standards. Whether informal logic should be seen as dialectical or not, in our opinion, needs to be treated as a separate issue, albeit an important one for further discussions.

The proof standards presented above and the weights assigned by the audience to the arguments can at least arguably be taken to represent a way of thinking characteristic of systems of legal reasoning, such as adopted in the common law. But there can also be a philosophical justification offered for supporting the use of standards of proof in epistemology. People who take this approach are generally categorized as qualified skeptics, who take the view that no claim can be proved beyond all doubt, even a claim based on an immediate perception, such as 'I now see a red light in front of my face'. On this skeptical view, what determines whether a claim is justified is the weighing of the pro and con arguments as evidence is collected and evaluated 
during a procedure of asking and answering critical questions. For acceptance of the claim to be rationally justified, the pro arguments have to be stronger than the con arguments against it to a degree stipulated at the opening stage of the inquiry. This degree of required strength for an argument to be a proof is called the standard of proof.

Carneades the ancient philosopher was a qualified skeptic who held the view that no argument is conclusive in the sense that it proves its conclusion beyond all doubt. As skeptical philosophers such as Carneades have long argued, even the argument "I now see a red light in front of my face, therefore there is a red light in front of my face" might turn out not to prove its conclusion beyond all doubt, even granting that its premises are accepted as true. For the qualified skeptic, there can be conclusive arguments, provided a conclusive argument is defined as one that meets its standard of proof, perhaps even the very high standard of beyond reasonable doubt. According to the qualified skeptic, this is the highest standard that can be met by real arguments. In other words, according to this viewpoint, a conclusive argument should not be defined as one that proves its conclusion beyond all doubt, for this is a standard of proof that fallible agents can never attain.

The proof standards modeled thus far in CAS do not compare the set of pro arguments against the set of con arguments, but rather only compare each pro argument against each con argument. Summing the weights of arguments to check if the sum of the weights of the pro arguments outweigh the sum of the weights of the con arguments only makes sense if the arguments are independent, to avoid double counting. CAS can be easily extended with further proof standards for comparing sets of pro and con arguments, but users would need to take responsibility to assure that these proof standards are used only when the arguments are independent.

These issues are discussed more thoroughly in (Gordon and Walton, 2009). More could be said about how to model Wellman's lawn-mowing example. For example we could put in an enthymeme stating that lawn-cutting would leave no time for movie-going, and so forth. But basically CAS can handle the pro-contra aspect, however you decide on the details or put in more information about what the propositions the audience accepts, how they weigh the arguments, and what proof standards are required. 


\section{Conclusion}

Have we proved that CAS allows for a representation of all informal logic-based arguments? Our answer is that we do not claim to have proved this, since not all of the ten characteristics have been successfully modeled, at least not in a way which would meet with broad consensus. This applies in particular to the characteristic of relevance. As stated at the beginning, our proof of progress towards the goal of formalizing informal logic is premised on the assumption that these ten characteristics are adequate. If one does not accept this assumption, one need not accept our claims. However, if you read our paper as a discussion of whether any formal system (particularly CAS) can fulfill the ten postulated requirements, it is much more interesting as a way forward to finding the relationship between formal models of argumentation of the kind currently being in artificial intelligence and informal logic as practical set of tools for helping users identify, analyse and evaluate real arguments of the kind all of us have to deal with every day in our professional work and education, and indeed in all daily life.

Reconstructing arguments found in a text of natural language discourse is an informal logic skill that often requires an ability to grasp all kinds of subtle nuances such as implicit premises and Gricean implicature. This kind of skill can be enhanced by teaching students to use such informal logic tools as argument diagramming and argumentation schemes. Using a computational tool such as CAS will not automatically analyze or evaluate arguments in natural language texts by itself (autonomously), replacing the need for such skills to be taught. But it can help users carry out such tasks of critically assessing arguments as (1) testing whether the argument supposedly identified in a natural language text fits an argumentation scheme, (2) finding implicit premises need to make the given argument fit the scheme, (3) asking appropriate critical questions matching this scheme, and (4) determining which claims are acceptable by using input concerning assumptions the audience presumably accepts.

Hence a formal argumentation system such as CAS is not an automated informal logic that can be mechanically applied to evaluate an argument without relying on the intuitions of a human user, or on using linguistic markers such as argument indicator words and the like. Nevertheless, as shown in this paper a formal and computational argumentation system such as CAS, because it has a well-defined logical structure that is applicable to the concepts and tasks characteristic of informal logic, does 
offer a formalization of informal logic. This has been proved by showing how the logical structure of CAS applies to key characteristics of informal logic as a working discipline designed to carry out specific tasks.

In this paper we formulated ten characteristics of informal logic, based on at least some of the literature that has attempted to set them out in an orderly and clear manner, and showed why they are identifiable with the discipline of informal logic as a school of thought and methodology for logic. We have made our case that CAS can model all of these characteristics within its formal structure. We do not claim that CAS is the only formal argumentation system that can formalize informal logic, but we also hope we have shown that it might have some advantages for doing it in a useful way that can be applied to "real" arguments. Even though in this paper we did not use CAS to model the argumentation in a fairly large real case, this work has already done elsewhere, for example in (Walton, 2013).

The weakest link in our chain of argumentation is our hypothesis that CAS can be used to model relevance. We admit this claim requires further research. According to Johnson $(2009,29)$ although there have been many attempts to develop a theory of relevance, none of them has been entirely successful. However, he also added (29) that sufficiency is the RSA criterion that has received the least attention, and that is where CAS is the strongest. We claim that a strong point of CAS is its use of proof standards to evaluate arguments. This move is unusual in logic and epistemology, fields that have long suffered from their failure to use proof burdens and standards to determine when defeasible argumentation can be closed off.

There remain some differences of opinion within the informal logic community on three key issues. One is how to define a conductive argument. A second one is whether conductive argument is essential for informal logic. In answer to an email query of mine (Sept. 12, 2012), Ralph Johnson agreed with the definition of conductive argument as evaluating argumentation by taking into account the arguments for some contested claim as well as the arguments against it, and weighing the one side against the other. He also agreed that this type of argument was characteristic of informal logic. Tony Blair (also on Sept. 12, 2012) had a different approach. He specified a conductive argument as one where the arguer has decided (or already determined) that the arguments for the claim in question are good reasons for accepting it, and has also decided that the arguments against the claim in question are good reasons for rejecting it, but none on either side is decisive, and the strength of the com- 
bined arguments for accepting the claim outweighs the strength of the combined arguments for rejecting it. He remarked that he didn't see a commitment to conductive arguments as essential for informal logic. These matters might be clarified in Blair's OSSA paper on conductive argument.

The third issue is whether informal logic is dialectical. CAS argument graphs are evaluated in stages of dialogue, as indicated in the last example. Modeling shifts in the burden of proof in real arguments, we have argued using the last example, is part of the process of rational argumentation in dialogues that in our opinion, should also be a characteristic of informal logic. However, there are some in the informal logic community, and very many in the formal logic and epistemology communities, who might disagree that evaluating an argument requires reference to a conversational (dialogue) setting. On this point there appears to be a difference of opinion in the informal logic community. Some accept dialogue structures as useful tools for informal logic methodology, while others appear reluctant to do so. As noted in section 8 , it is unclear to us how the issue of the role of dialogue in informal logic is related to the issue of whether or not informal logic can be formalized. These need to be treated as separate (orthogonal) issues for the purpose of this paper, given that we have shown that informal logic can be formalized, no matter what position one takes on the issue of the role of dialogue in informal logic.

Acknowledgements: Both authors would like thank the Social Sciences and Humanities Research Council of Canada for Insight Grant 435-2012-0104 that supported the work in this paper.

\section{References}

Adler, J.E. (2013). Are conductive arguments possible? Argumentation 27, 245-257.

Atkinson, K., Bench-Capon, T. J. M. and McBurney, P. (2006). Computational Representation of Practical Argument, Synthese, 152 (2), 157-206.

Ballnat, S. and Gordon, T. F. (2010). Goal Selection in Argumentation Processes, Computational Models of Argument: Proceedings of COMMA 2010, ed. P. Baroni, F. Cerutti, M. Giacomin and G. R. Simari, Amsterdam, IOS Press, 51-62. 
Besnard, P., Garcia, A., Hunter, A., Modgil, S., Prakken, H., Simari, G. and Toni, F. (2014), Introduction to Structured Argumentation, Argument \& Computation, 5(1), 1-4. DOI: 10.1080/19462166.2013.869764

Barth, E. M. and Krabbe, E. C. W. (1982). From Axiom to Dialogue. Berlin: de Gruyter.

Bin W. \& Prakken, H. (2012). Determining the Structure of Arguments with AI Models of Argumentation. Proceedings ECAI-12 Workshop on Computational Models of Natural Argument. Montpellier, France, 2012, to appear with Springer.

Blair, J. A. (2001). Walton's Argumentation Schemes for Presumptive Reasoning: A Critique and Development, Argumentation, 5, 365-379.

Blair, J. A. (2012). Groundwork in the Theory of Argumentation. Dordrecht: Springer.

Blair, J.A. (2013). Are conductive arguments really not possible? In Virtues of Argumentation. Proceedings of the 10th international conference of the Ontario Society for the Study of Argumentation (OSSA), 22-26 May 2013, ed. D. Mohammed and M. Lewiński, 1-13. Windsor, ON: OSSA.

Blair, J. A. and Johnson, R. H. eds. (2011). Conductive Argument: An Overlooked Type of Defeasible Reasoning. London: College Publications.

Copi I. and Cohen, C. (1990). Introduction to Logic, $8^{\text {th }}$ ed. New York: Macmillan.

Dung, P. (1995).On the Acceptability of Arguments and its Fundamental Role in Nonmonotonic Reasoning, Logic Programming and n-person Games, Artificial Intelligence, 77(2), 321-357.

Freeman, J. B. (1998). What Sorts of Statements Are There? A Philosophical Look at Stasis Theory, Argumentation \& Rhetoric. Argumentation \& Rhetoric. CD-Rom. Edited and produced by Hans V. Hansen and Athena Colman. St. Catharines, Ontario. OSSA, August, 1998, 1-15. Downloaded from this site on January 26, 2015:

http://scholar.uwindsor.ca/cgi/viewcontent.cgi?article $=1883$ \&context $=$ ossaarchive

Gordon, T. F. (2010). The Carneades Argumentation Support System, Dialectics, Dialogue and Argumentation, ed. C. Reed and C. W. Tindale, London: College Publications.

Gordon T. F. and Walton, D. (2006). The Carneades Argumentation Framework, Computational Models of Argument: Proceedings of COMMA 2006, ed. P. E. Dunne and T. J. M. Bench-Capon. Amsterdam: IOS Press, 195-207. 
Gordon, T. F., H. Prakken, and D. Walton (2007). The Carneades Model of Argument and Burden of Proof, Artificial Intelligence 171: 875-96.

Gordon, T. F., and Walton, D. (2009). Proof Burdens and Standards. In Argumentation in Artificial Intelligence, I. Rahwan and G. Simari, Eds. Springer-Verlag, Berlin, Germany, 2009, 239-260.

Gordon, T. F., and Walton, D. (2011). A Formal Model of Legal Proof Standards and Burdens. In 7th Conference on Argumentation of the International Society for the Study of Argumentation (ISSA 2010) (Amsterdam, 2011), F. van Eemeren, B. Garssen, J. A. Blair, and G. R. Mitchell, Eds., no. 1969, Sic Sac, 644-655.

Hohmann, H. (1989). The Dynamics of Stasis: Classical Rhetorical Theory and Modern Legal Argumentation, American Journal of Jurisprudence, 34, 1989, 171-197.

Johnson, R. H. (2006). Making Sense of Informal Logic, Informal Logic, 26(3), 231-258.

Johnson, R. H. (2009). Some Reflections on the Informal Logic Initiatives, Studies in Logic, Grammar and Rhetoric, 16 (29), 17-46.

Johnson, R.H. and J.A. Blair. (1977). Logical Self-Defense. Toronto: McGraw-Hill Ryerson.

Kienpointner, M. (1997). On the Art of Finding Arguments: What Ancient and Modern Masters of Invention Have to Tell Us about the Ars Inveniendi, Argumentation, 11 (2), 225-236.

McFadden, P. M. (1988). The Balancing Test, Boston College Law Review, 29(3), 585-656.

S.J. Modgil \& H. Prakken (2014). The ASPIC+ Framework for Structured Argumentation: a Tutorial. Argument and Computation, 5, 31-62.

http://www.cs.uu.nl/groups/IS/archive/henry/ASPICtutorial.p $\underline{\mathrm{df}}$

Pollock, J. L. (1995). Cognitive Carpentry. Cambridge, Mass.: The MIT Press.

Prakken, H. (2010). An Abstract Framework for Argumentation with Structured Arguments. Argument and Computation 1, 93-124.

Prakken, H. (2011). An Overview of Formal models of Argumentation and their Application in Philosophy. Studies in Logic, 4(1), (2011):65-86.

http://www.cs.uu.nl/groups/IS/archive/henry/china11.pdf 
Scheuer, O., Loll, F., Pinkwart, N. and McLaren, B. M. (2010). Computer-supported Argumentation: A Review of the State of the Art, Computer-Supported Collaborative Learning, 5 (1), 43-102.

Tindale, C. W. (1999). Acts of Arguing: A Rhetorical Model of Argument. Albany: State University of New York Press.

Verheij, B. (2003). Dialectical Argumentation with Argumentation Schemes: An Approach to Legal Logic, Artificial Intelligence and Law, 11, 167-195.

Verheij, B. (2005). Virtual Arguments. On the Design of Argument Assistants for Lawyers and Other Arguers. The Hague: TMC Asser Press.

Walton, D. (1996). Argument Structure: A Pragmatic Theory. Toronto: University of Toronto Press.

Walton, D. (2004). Relevance in Argumentation. Mahwah, New Jersey: Lawrence Erlbaum Associates.

Walton, D. and Gordon T. F. (2012). The Carneades Model of Argument Invention, Pragmatics \& Cognition, 20(1), 1-31.

Wellman, C. (1971). Challenge and Response: Justification in Ethics, Carbondale, Southern Illinois University Press, 1971. 